\title{
Palabres autour des arbres : des discours sur leur intelligence aux dérives de l'anthropomorphisme
}

\author{
Jean-Louis Doucet
}

Université de Liège - Gembloux Agro-Bio Tech. TERRA. Forest is Life. Passage des Déportés, 2. BE-5030 Gembloux (Belgique).E-mail : jldoucet@uliege.be

Reçu le 20 avril 2020, accepté le 10 juin 2020, mis en ligne le 2 juillet 2020.

Cet article est distribué suivant les termes et les conditions de la licence CC-BY (http://creativecommons.org/licenses/by/4.0/ deed.fr)

Le présent document constitue la synthèse de la leçon inaugurale présentée en septembre 2019 à l'Université de Liège Gembloux Agro-Bio Tech dont le texte entier est présenté en annexe.

L'intelligence des arbres est un sujet qui passionne les populations urbaines. Elle fait l'objet d'un débat animé entre les différents acteurs du secteur forestier. La présente note vise à objectiver ce débat en synthétisant les connaissances récentes sur le sujet. Elle montre que les arbres peuvent communiquer entre eux, soit via des molécules volatiles, soit via des échanges souterrains, notamment grâce à un réseau mycorhizien. Les arbres peuvent aussi percevoir la lumière et les sons. Ils peuvent donc s'adapter à un environnement changeant, ce qui traduit une certaine forme d'intelligence. Néanmoins, renoncer à l'exploitation du bois sur cette base ne peut être justifié. Cela se traduirait par des impacts environnementaux dont la portée est occultée par les détracteurs de l'exploitation forestière.

Mots-clés. Arbre, intelligence, communication, composé volatil, mycorhizé, exploitation forestière, déboisement.

\section{Palaver around the trees: discussions about their intelligence to the drifts of anthropomorphism}

The intelligence of trees is an issue that fascinates people in urban areas. It is the subject of passionate debate between forest sector stakeholders. This article aims to objectify this debate by synthesizing recent knowledge on the subject. It shows that trees can communicate with each other, either via volatile molecules or via underground exchanges, in particular through a mycorrhizal network. Trees can also perceive light and sound. They can therefore adapt to a changing environment, which reflects a certain form of intelligence. Nevertheless, abandoning timber logging for this reason is not acceptable. This would result in environmental impacts that are often overlooked by the opponents of logging.

Keywords. Trees, intelligence, communication, volatile compounds, mycorrhizae, logging, deforestation.

\section{INTRODUCTION}

Dans les régions urbanisées, un nouveau rapport à l'arbre s'exprime: l'arbre y est perçu comme une source d'apaisement et de retour au sacré. Le livre $L a$ vie secrète des arbres de Wohlleben (2017) a largement contribué à ce nouveau paradigme. Son immense succès est lié à l'approche empathique de l'auteur envers les arbres auxquels il attribue sensibilité, mémoire et intentions. De nombreux chercheurs ont critiqué l'ouvrage, considérant qu'il reposait sur un nombre limité de sources scientifiques, alors que d'autres considèrent l'approche utile pour pouvoir vulgariser les découvertes sur les aptitudes des plantes à communiquer (Teyssèdre, 2018).

La présente note, issue de la leçon inaugurale de rentrée académique de Gembloux Agro-Bio Tech 2019-
2020, a pour objet de faire le point sur « l'intelligence » des arbres tout en soulignant les dangers liés à l'approche anthropomorphique. Ceux-ci sont toutefois davantage expliqués dans le texte intégral repris en annexe. La revue de littérature n'a pas pour ambition d'être exhaustive, elle repose sur une sélection de publications de référence dans le domaine.

\section{LITTÉRATURE SUR L'INTELLIGENCE DES ARBRES}

Les premières études sur les talking trees (Baldwin \& Schultz, 1983) ont suscité de nombreuses controverses (voir par exemple Fowler \& Lawton, 1985) en raison des faiblesses statistiques liées à l'absence de répétitions indépendantes. Toutefois, des travaux ultérieurs, 
menés cette fois avec des approches statistiquement robustes et plusieurs modèles biologiques, plaident en faveur d'une réelle communication entre les plantes, notamment via les molécules volatiles.

Karban et al. (2014) ont réalisé une méta-analyse afin d'objectiver le rôle de ces molécules dans la lutte contre l'herbivorie. Quatre-vingt-trois pour cent des 48 travaux retenus ont rapporté que l'herbivorie était réduite chez les plantes qui étaient exposées à des molécules volatiles émises en réponse à l'attaque d'un agresseur. Selon Heil (2014), les « récepteurs » des composés organiques volatils sont nombreux : les parties éloignées d'une même plante, les plantes voisines, les herbivores et leurs prédateurs. Les plantes peuvent en effet produire des substances répulsives envers les agresseurs ou attractives vis-à-vis de leurs prédateurs ou parasites (Aljbory \& Chen, 2018). Par ailleurs, les molécules volatiles peuvent aussi intervenir dans l'attraction des pollinisateurs (Kantsa et al., 2019) ou dans la dispersion de graines via la zoochorie (par exemple Tosso et al., 2018) (Figure 1).

Le transfert souterrain de molécules entre les arbres est un autre sujet de plus en plus documenté. Il y a une cinquantaine d'années, sous les tropiques, Leroy-Deval (1973) mettait en évidence des soudures racinaires entre des okoumés (Aucoumea klaineana Pierre) appartenant à des strates de végétation différentes. Les anastomoses racinaires permettraient aux individus les plus faibles de subsister grâce au flux de substances nutritives provenant des arbres vigoureux de la canopée. En retour, les dominants bénéficieraient d'une extension de leur rhizosphère. Bien que le transfert de substances nutritives entre individus n'ait pas été prouvé, il demeure probable étant donné l'observation fréquente de souches d'okoumé demeurant vivantes

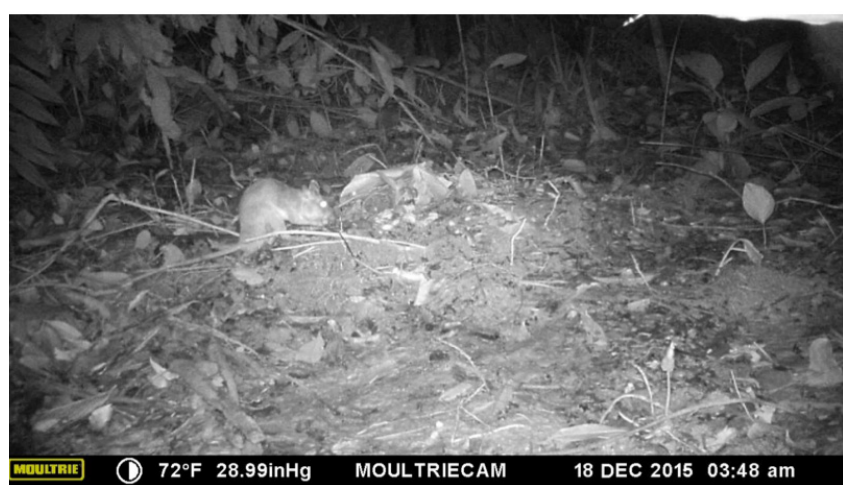

Figure 1. Un rat d'Emin (Cricetomys emini Wroughton, 1910) attiré par la sulcatone (6-méthyl-5-heptène-2-one) émise par l'arille de la graine de Guibourtia tessmannii (Harms) J.Léonard - A giant pouched rat (Cricetomys emini Wroughton, 1910) attracted to sulcatone (6-methyl-5hepten-2-one) emitted by the aril of the seed of Guibourtia tessmannii (Harms) J.Leonard. Source : Tosso et al., 2018. de nombreuses années après la disparition du tronc (Figure 2).

En plus, ou à la place, d'un transfert direct, les mycorhizes semblent jouer un rôle déterminant dans la communication entre arbres. La plante hôte et les champignons associés forment un partenariat: les hyphes du mycélium fournissent des nutriments et de l'eau en échange des produits issus de la photosynthèse (Chaudhary et al., 2016). Selon Simard (2018), les réseaux de champignons mycorhiziens relient les racines de plusieurs arbres et facilitent les échanges de ressources et de moyens de défense. Ils permettraient même de reconnaitre des parents. L'auteur considère que l'organisation d'un réseau mycorhizien dans un peuplement forestier, où les arbres sont des nœuds et les hyphes des liens, est très similaire à l'organisation d'un réseau neuronal. Certains acides aminés ou phytohormones transmises par le réseau sont analogues à des neurotransmetteurs et seraient responsables de réactions d'apprentissage, voire de mémorisation. Leur ampleur serait suffisante pour influencer l'absorption racinaire, l'assimilation de nutriments, la croissance et la survie de la plante

D'autres caractéristiques peuvent être mises en évidence pour suggérer une certaine forme d'intelligence chez les végétaux. Les arbres bougent, mais très lentement, car ils cherchent la lumière et la verticalité. Pour se maintenir droit, ils ont des capteurs dans leurs cellules permettant de détecter l'inclinaison et de se redresser (Pouliquen et al., 2017). Les arbres perçoivent les variations de lumière et y répondent. Au moins cinq groupes de photorécepteurs reçoivent des informations et permettent aux plantes d'entrer ou de sortir de dormance, d'accélérer ou d'arrêter la croissance, de favoriser ou de retarder la floraison, d'adapter la direction de la croissance, d'induire ou de supprimer la formation de pousses latérales, etc. (Voitsekhovskaja, 2019). Enfin, les arbres percevraient

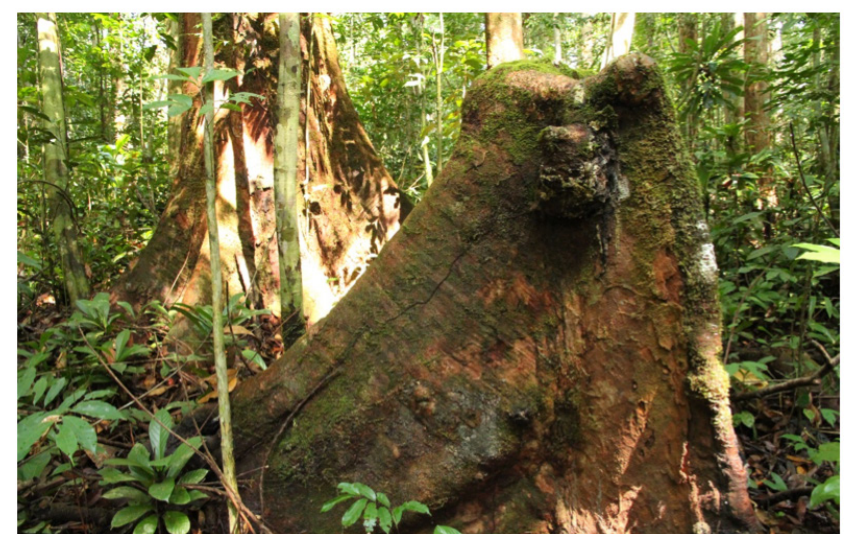

Figure 2. Souche d'okoumé vivante malgré l'absence de tronc - A stump of okoumé (gaboon) still alive despite the absence of a trunk. (C) J.-L. Doucet 
des sons. Il a été démontré récemment que les fleurs pouvaient distinguer des vibrations induites par des pollinisateurs et adapter en conséquence la qualité du nectar. Cette perception serait rendue possible grâce à la vibration des pétales, laquelle serait perçue par des mécanorécepteurs (Veits et al., 2019).

\section{DISCUSSION SUR LES DÉRIVES DE L'ANTHROPOMORPHISME}

En considérant ce qui précède, on pourrait considérer que les arbres sont effectivement intelligents, si l'intelligence est «un ensemble de processus qui permet d'apprendre ou de s'adapter à des situations nouvelles » (Fournier \& Moulia, 2008). Mais le sontils plus que les plantes herbacées sur lesquelles ont été conduites la plupart des expérimentations sur le sujet ? La question n'est ni anodine, ni sans conséquences car selon Peter Wohlleben (2017): "Quand on sait qu'un arbre est sensible à la douleur et a une mémoire, que des parents-arbres vivent avec leurs enfants, on ne peut plus les abattre sans réfléchir ni ravager leur environnement en lançant des bulldozers à l'assaut du sous-bois ».

En vertu d'une symbolique forte (Boudon, 2010), les arbres sont bien plus idéalisés que d'autres végétaux non ligneux. Alors que le rôle alimentaire de ces derniers n'est pas remis en question, Wohlleben (2017) considère que «nous devons traiter les arbres comme nous traitons les animaux en leur évitant des souffrances inutiles ». Cette prise de position pourrait se traduire chez certains par le boycott du bois et un plaidoyer contre l'exploitation forestière. Or renoncer à celle-ci pourrait avoir des conséquences environnementales qu'il convient de ne pas négliger. D'une part, dans les pays du Nord, cela se traduirait par l'utilisation d'autres matériaux plus énergivores et polluants. D'autre part, dans les pays du Sud, renoncer à l'exploitation forestière sélective priverait ces pays des devises indispensables à leur développement et in fine accélèrerait la conversion des forêts en terres agricoles, première cause mondiale de déforestation (Curtis et al., 2018).

\section{CONCLUSIONS}

La vulgarisation à l'extrême des nombreux travaux sur la communication entre les arbres et l'approche très anthropomorphique de Wohlleben (2017) ont suscité des débats animés entre les différentes catégories d'acteurs du secteur forestier. Interpellés par les médias, les chercheurs ont eu une opportunité unique d'expliquer leurs travaux sur l'intelligence des arbres. Il convient toutefois de ne pas surinterpréter cette intelligence et de ne pas adopter des attitudes excessives qui finalement occulteraient la fonction de production des écosystèmes forestiers, laquelle est indispensable au développement durable de nos sociétés dans un contexte de changement global.

\section{Remerciements}

L'auteur remercie chaleureusement toutes les personnes qui ont contribué à améliorer le texte d'allocution de rentrée académique : J. Hébert, G. Lognay, A. Fayolle, C. Vermeulen, P. Lejeune, H. Claessens, G. Ligot, J-Y. De Vleeschouwer, C. Geerts, C. Laurent, J. Challe, P. Maesen, L. Tanaskovic, M-L. Fauconnier.

\section{Bibliographie}

Aljbory Z. \& Chen M.S., 2018. Indirect plant defense against insect herbivores: a review. Insect Sci., 25, 2-23, doi.org/10.1111/1744-7917.12436

Baldwin I.T. \& Schultz J.C., 1983. Rapid changes in tree leaf chemistry induced by damage: evidence for communication between plants. Science, 221, 277-279, doi.org/10.1126/science.221.4607.277

Boudon B., 2010. Symbolisme de l'arbre. Paris: Les Éditions du Huitième Jour.

Chaudhary V.B. et al., 2016. MycoDB, a global database of plant response to mycorrhizal fungi. Sci. Data, 3, 1-10, doi.org/10.1038/sdata.2016.28

Curtis P.G. et al., 2018. Classifying drivers of global forest loss. Science, 361, 1108-1111, doi.org/10.1126/science. aau3445

Fournier M.\&Moulia B.,2008.Sensibilitéetcommunication des arbres : entre faits scientifiques et gentil conte de fée. For. Wallonne, 149, 12-21.

Fowler S.V. \& Lawton J.H., 1985. Rapidly induced defenses and talking trees: the devil's advocate position. Am. Nat., 126, 181-195, doi.org/10.1086/284408

Heil M., 2014. Herbivore-induced plant volatiles: targets, perception and unanswered questions. New Phytol., 204, 297-306, doi.org/10.1111/nph.12977

Kantsa A. et al., 2019. Floral volatiles and visitors: a metanetwork of associations in a natural community. J. Ecol., 107, 2574-2586, doi.org/10.1111/1365-2745.13197

Karban R., Yang L.H. \& Edwards K.F., 2014. Volatile communication between plants that affects herbivory: a meta-analysis. Ecol. Lett., 17, 44-52, doi.org/10.1111/ ele. 12205

Leroy-Deval J., 1973. Les liaisons et anastomoses racinaires. Bois For. Trop., 1(52), 37-49.

Pouliquen O. et al., 2017. A new scenario for gravity detection in plants: the position sensor hypothesis. Phys. Biol., 14, 1-13, doi.org/10.1088/1478-3975/aa6876.

Simard S.W., 2018. Mycorrhizal networks facilitate tree communication, learning, and memory. In: Baluska F., Gagliano M. \& Witzany G., eds. Memory and learning 
in plants. Springer International Publishing, 191-213, doi.org/10.1007/978-3-319-75596-0

Teyssèdre A., 2018. Trois regards sur le livre de Peter Wohlleben "La vie secrète des arbres », https://www. sfecologie.org/regard/ro5-mars-2018/, (17/04/2020).

Tosso F. et al., 2018. Characterization of animal communities involved in seed dispersal and predation of Guibourtia tessmannii (Harms) J. Léonard, a species newly listed on Appendix II of CITES. Afr. J. Ecol., 56, 468-476, doi. org/10.1111/aje.12480
Veits M. et al., 2019. Flowers respond to pollinator sound within minutes by increasing nectar sugar concentration. Ecol. Lett., 22, 1483-1492, doi.org/10.1111/ele.13331

Voitsekhovskaja O.V.,2019.Phytochromes and other (photo) receptors of information in plants. Russ. J. Plant Physiol., 66, 351-364, doi.org/10.1134/s1021443719030154

Wohlleben P., 2017. La vie secrète des arbres. Paris: Éditions des Arènes.

(18 réf.) 